\title{
Design of Novel Energy Recovery Damper Based on EAP Zhen-Tao WANG ${ }^{1, a}$, Jian-Bo $C^{1, b_{*}}$, Shi-Ju $E^{1, b}$, Tian-Feng ZHAO ${ }^{2, a}$, Can YANG $^{1, b}$, Zhao GAO ${ }^{1, b}$, Han-Pin LUO ${ }^{1, b}$ and Zhong-Yao WU ${ }^{1, a}$ \\ ${ }^{1}$ College of Engineering, Zhejiang Normal University, Jinhua, Zhejiang 321004, P.R. China \\ ${ }^{2}$ Library, Zhejiang Normal University, Jinhua, Zhejiang 321004, P.R. China \\ a374944227@qq.com, ${ }^{\text {b*C} C o r r e s p o n d i n g ~ a u t h o r: ~ c a o j i a n b o 2008 @ 163 . c o m ~}$
}

Keywords: Damper, Energy recovery, Electroactive polymer, Vibration generation

\begin{abstract}
Damper is an important part of vehicle suspension system, containing the nonlinear vibration system of elastic force and damping force, this system generates vibration excited by road irregularities and vibration source such as engine. To solve shortcomings of the traditional vehicle that consumes energy high and wastes the vibration energy, in this paper, through analyzing the existing principle of vehicle vibration energy recovery and power generation mechanism of electroactive polymer (EAP), integrating both advantages, design a novel energy recovery damper based on EAP, draw three-dimensional maps and two-dimensional maps of novel energy recovery damper and suspension system with Solidworks software, and do the simulation study about the vibration power generation process of this damper. It can be achieved to recycle the car body vibration energy through the vibration energy recovery damper, translate the kinetic energy of the car body vibration into electrical energy to charge the vehicle battery, so as to reduce the waste of energy, save energy and improve energy efficiency.
\end{abstract}

\section{Introduction}

Nowadays, the car has been the most important means of transportation. And energy saving as the current car design (especially the design of electric vehicle) is one of the most important problems in addition to improve the materials structure, storage technology and driving strategy, the current solution is to find a new way of energy regeneration. There are mainly two parts of the energy that vehicles can recycle: vibration energy recovery and braking energy recovery, which has been relatively mature, but when the vehicle is driving on uneven road, the vibration energy that excited by the road didn't cause the concern and use of people usually translates into heat energy by the vehicle dampers and then dissipates.

EAP is a novel EAP materials based on Maxwell effect. Compared with other types of EAP, EAP has many characteristics such as better flexibility, producing greater strain, having low density and low cost, etc [1-4]. This material can be used as the driver material, as the bionic muscles and related devices that are used for structuring MEMS, such as micro motor, micro pump, micro valve, etc; and can be used for power generation, especially applying to distributed generation and other occasions $[5,6]$.

In this paper, based on researching technologies such as the principle of the vibration generation and the energy recovery and utilization, a novel energy recovery damper was designed with electroactive polymer (electroactive polymers, EAP). It can be achieved to recycle the car body vibration energy through the vibration energy recovery damper. The study provides some technical basis so that the EAP materials would be widely used in the field of vibration energy recovery, and can solve the above problem of the vibration energy waste better.

\section{Generation Principle of EAP}

EAP is formed by painting on the surface of the acrylic elastomer matrix with electrode materials. It can be regarded as a variable capacitor that the sandwich structure is composed of the thin passive elastomer that sandwiched between two compliant electrodes. 
EAP applies an electric field on the rubber insulator through two flexible electrodes, when the voltage is applied, the incompressible polymers would become thinner in the thickness and larger in the area. When the voltage is removed, the polymer would become thicker in the thickness and smaller in the area. So, EAP is like a variable capacitance electric generator. As shown in Fig. 1, when the EAP is stretched, elastomer films will increase in the area decrease in the thickness to store energy, when the EAP is contractive and relaxing, to achieve electricity-generation [7-10].

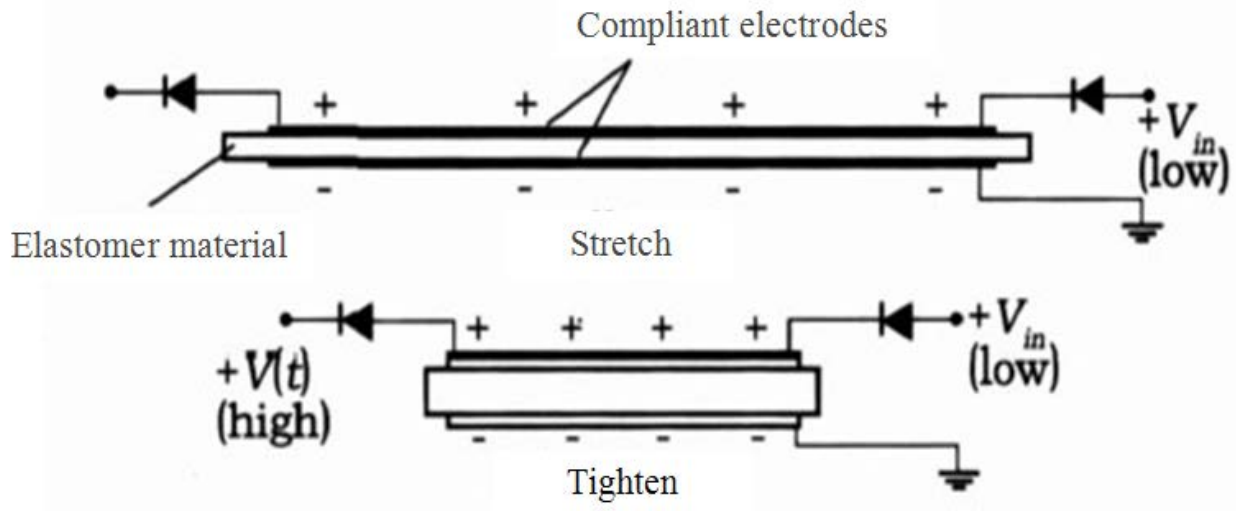

Fig. 1 Schematic diagram of EAP generation

According to the electricity-generation principle of dielectric elastomer, it can be simplified for the variable capacitance device, so the capacitance $C$ is:

$$
C=\varepsilon \varepsilon_{0} A / d
$$

where $\varepsilon$ was the relative dielectric constant; $\varepsilon_{0}$ was the permittivity of free space, is a constant, $\varepsilon_{0}=8.85 \times 10^{-12} \mathrm{~F} / \mathrm{m}$; $A$ was the area of EAP materials; $d$ was the thickness of EAP materials.

The area $A$ and thickness $d$ changed with changes of the materials. Where $B$ denotes the volume of the material, have:

$$
B=A d
$$

When (2) was substituted into the formula (1), have:

$$
C=\varepsilon \varepsilon_{0} A^{2} / B
$$

At any time, the charge voltage in the EAP film $V$ can be expressed as:

$$
V=\frac{Q}{C}=\frac{Q B}{\varepsilon \varepsilon_{0} A^{2}}
$$

where $Q$ was the charge on the film of EAP material.

For a single cycle of extension and contraction, the output electricity-generation energy of EAP $E$ was related to the change of EAP capacitance, was expressed as:

$$
E=\eta \varepsilon \varepsilon_{0} B E_{\max }^{2} \ln \left[C_{i} / C_{f}\right]
$$

where $C_{i}$ and $C_{f}$ are the total capacitance when the EAP layer was in the condition of expansion and contraction, respectively; $E_{\max }$ was the maximum electric field of EAP; $\eta$ was the conversion 
efficiency of the electric energy, including the conversion efficiency of electronics and the electromechanical coupling of materials. In conclusion, just know the change of the area of EAP in a deformation period (expansion and contraction), can calculate the size of the electric energy output.

\section{Design of Cylindrical EAP Energy Recovery Damper}

In this paper, the novel cylindrical damper that was designed includes cylindrical damper base, film, film rolling ring, the shell of dielectric elastomer damper, shell rolling ring, piston, spring, bolts, nuts and other basic components. The damper was designed in the form of the pneumatic type, and the back-and-forth simple harmonic motion with the spring seat movement was done in the way of the piston motion. The 3D design of cylindrical damper was shown in the following Fig. 2.

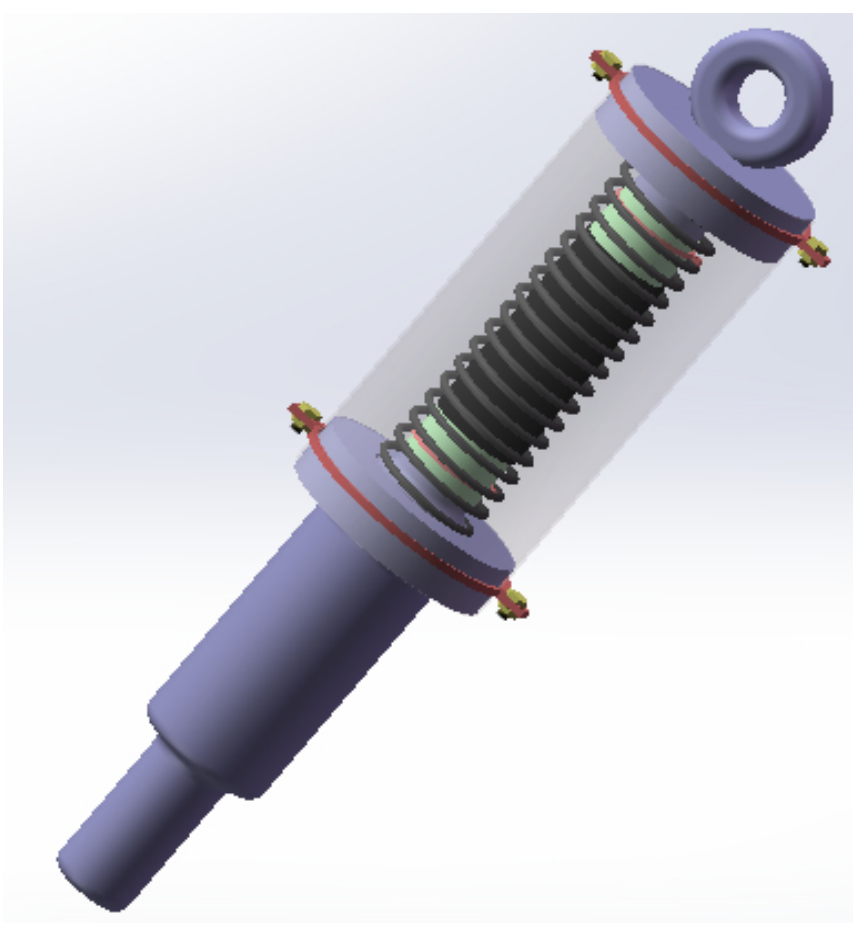

Fig. 2 The 3D design of cylindrical damper

The green part that shown in the Fig. 2 was film and the black coating on the film was the electronic coating, the expanded form of the film was rectangle, close to both ends of the base, and fasten with tie. When the internal part of damper did the back-and-forth movement, the upper and lower layers of new materials were painted with the electronic coating, and continued to do the movement of expansion and contraction, thus the damper realized the function of charging and discharging.

The novel material was fasten on the base of the cylindrical damper with film ties, and ligature another layer of dielectric elastomers in order to prevent the leakage phenomenon of damper during the electricity-generation. The degrees of tension and compression of the elastomer and spring were basically in the same, as a result, there was good coordination in the process of the movement of the spring.

General Assembly of Cylindrical Damper. The general assembly of cylindrical damper was showed in Fig. 3.

Parts of Cylindrical Damper. In this paper, the base of the cylindrical damper, piston and other organizations among the parts of the cylindrical damper were made by SC45C steel, the film material was made by EAP elastomer, the spring were made by A7075-T6 aluminum alloy, and the shell of damper were made by the dielectric elastomer. The specific material and its property will be 
analyzed in the following. The key parts drawing of the cylindrical energy-recovery damper was shown in Fig. 4.

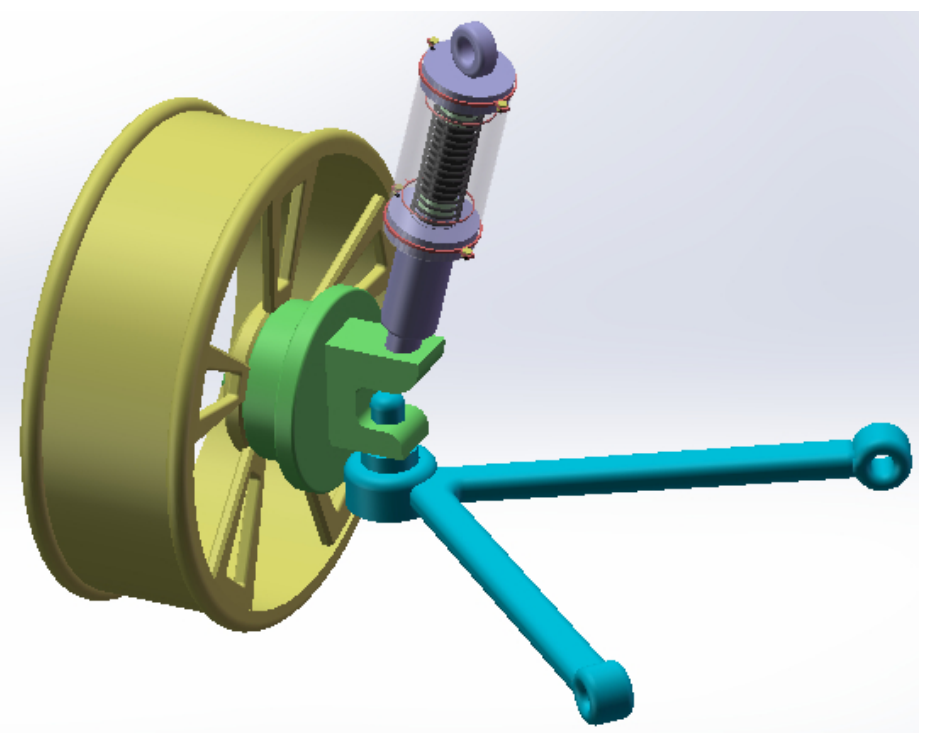

Fig. 3 The final rendering of cylindrical damper
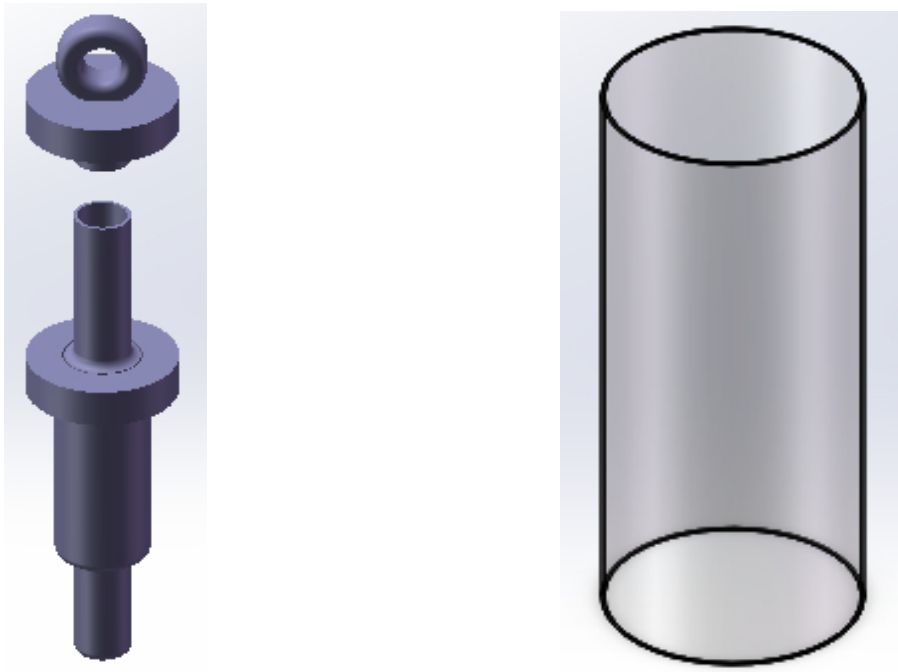

(a) The base of cylindrical damper

(b) Insulating elastomer damping

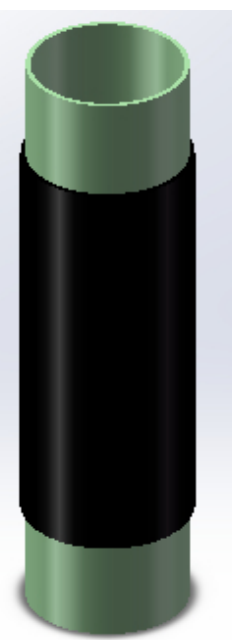

(c) Film

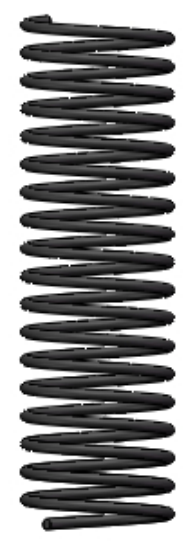

(d) Spring

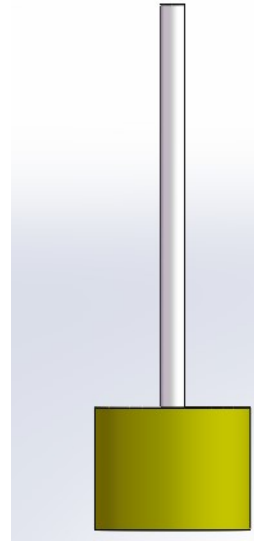

(e) Piston

Fig. 4 The key part drawings of cylindrical damper 


\section{Dynamic Simulation of Novel Cylindrical Damper}

The dynamic simulation about the vibration process of the novel cylindrical damper was done by Solidworks software, the simulation process of the expansion and contraction of the cylindrical damper was showed in Fig. 5. Add an axial force at the one end of the damper, with this force, the spring and EAP films of the internal damper and the dielectric elastomers of the external damper will be compressed, so the total damper will be compressed axially. At this point, the EAP material of internal damper would gather energy due to compression, when the force was removed, the damper returned to the normal from the state of compression, then change to the state of expansion. In the state of expansion, the spring and EAP films of the internal damper and the dielectric elastomers of the external damper would extend, so the total damper will be extended axially. The above was the total vibration electricity-generation of the novel cylindrical damper.

Fig. 5 (a) (b) represented the views when the damper was put flatwise and sidelong, and three shots of each picture represented the conditions of damper in the states of compression, normal and expansion, respectively.
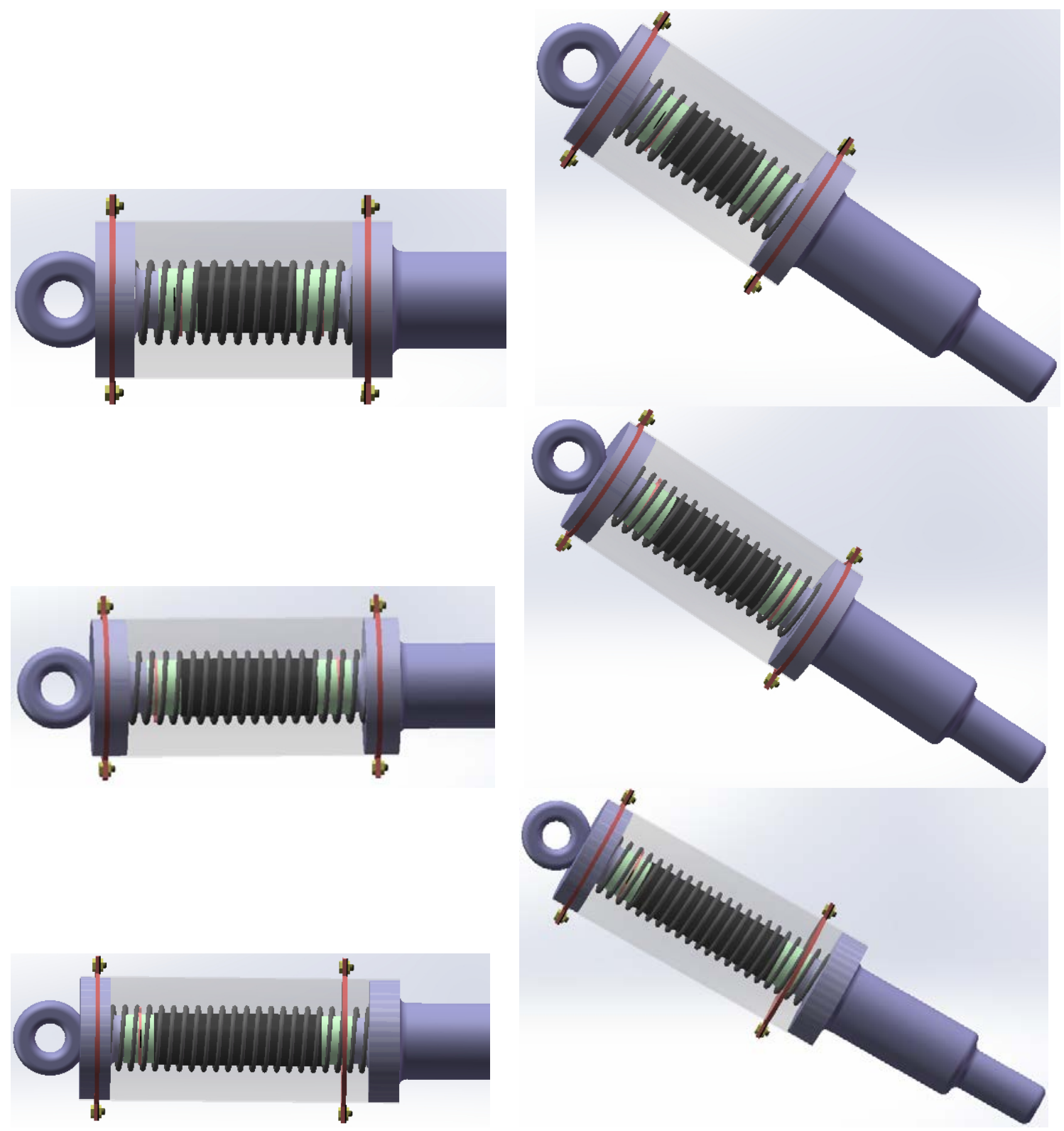

(a) The flat view of the dynamic simulation of cylindrical damper

(b) The sidelong view of the dynamic simulation of cylindrical damper

Fig. 5 The simulation process of the compression and expansion of cylindrical damper 


\section{Conclusion}

Based on analyzing the principle of vehicle vibration energy recovery and power generation mechanism of EAP, a novel energy recovery damper was designed with EAP. The threedimensional and two-dimensional maps of novel energy recovery damper were drawn by Solidworks software, and the simulation about the vibration power generation process of this damper was studied. The novel energy recovery damper has the functions of recycling vibration energy and generating electricity itself. Renewable energy, green environmental protection, energy conservation and other words have become a trend of the development of modern society. With the progress of science and technology, new materials are used to improve the existing structure of the damper, and it has good value to study a novel energy recovery damper based on EAP.

\section{Acknowledgement}

The work was supported by the National Natural Science Foundation of China (No. 51377146, 51407162), Zhejiang Provincial Natural Science Foundation of China (No. LQ13E050009), the 2013 Foundation of Education Department of Zhejiang Province of China (No. Y201329556).

\section{References}

[1] S.J. E, X.L. Zhu and C.F. Gao: Modern Manufacturing Engineering, (2009) No. 9, pp. 41-45.

[2] S.J. E, X.L. Zhu and J.B. Cao: Transactions of Agricultural Machinery, Vol. 41 (2010) No. 9, pp. 194-198.

[3] T.H. Tang: Application of Power Supply Technology, Vol. 10 (2007) No. 2, pp. 60-64.

[4] F.L. Gui, S.Q. Liu, L.H. Li and D. Li: Journal of Zhejiang Normal University (Natural Sciences), Vol. 38 (2015) No. 1, pp. 52-57.

[5] K.Q. Li, H.M. Wang and P.H. Liu: Electrical Technology and Automation, Vol. 40 (2011) No. 6, pp. 171-174.

[6] Q. Wei and H.L. Chen: Sensor World, Vol. 13(2007) No. 4, pp. 1-5.

[7] S.J. E and J.B. Cao: International Journal of Applied Electromagnetics and Mechanics, Vol. 36 (2011) No. 3, pp. 267-278.

[8] J.B. Cao, C,J. Ge, S.J. E, A.F. Liu, L.L. Jin and X.Q. Jiang: Transactions of the Chinese Society for Agricultural Machinery, Vol. 47 (2016) No. 3, pp. 389-394.

[9] Y.Q. Cai, J.B. Cao, Z.Y. Liu, S.J. E, Z.Y. Wu and H.B. Zhou: Key Engineering Materials, Vol. 620 (2014), pp. 312-317.

[10] Z.Y. Wu, J.B. Cao, S.J. E, T.F. Zhao, C.X. Chen, C.H. Yang and H.H. Bao: Key Engineering Materials, Vol. 579-580 (2013), pp. 349-352. 